\title{
Aqueous and ethanolic extracts of welsh onion, Allium fistulosum, attenuate high-fat diet-induced obesity
}

\author{
Yoon-Young Sung ${ }^{1}$, Dong-Seon $\mathrm{Kim}^{2}$, Seung-Hyung Kim³ and Ho Kyoung Kim ${ }^{1^{*}}$
}

\begin{abstract}
Background: Allium fistulosum (Welsh onion) is a traditional medicinal plant used for the treatment of colds, influenza, abdominal pain, headache, and heart disease. This study evaluated the effects of A. fistulosum ethanolic extract (AFE) and aqueous extract (AFW) on body weight and other obesity-related parameters.

Methods: Male 8-week-old C57BL/6 J mice were fed either a standard chow diet (normal control) or a high-fat diet (HFD) either alone (HFD-control) or in combination with G. cambogia extract containing hydroxycitric acid (HCA, an herbal weight-loss supplement), conjugated linoleic acid (CLA, a weight-loss supplement), orlistat (a clinically available anti-obesity drug), AFW, or AFE ( $n=6$ mice per group) for 6 weeks. At the end of 6 weeks, several body weight and obesity-related parameters were examined, including: liver and adipose weight, adipocyte size, serum lipid profiles, liver expression of adenosine monophosphate-activated protein kinase (AMPK), and adipose tissue expression of uncoupling protein 2 (UCP2).
\end{abstract}

Results: High-performance liquid chromatography showed that both AFE and AFW contain ferulic acid and quercetin. Oral administration of AFW and AFE to HFD-fed mice decreased body weight as well as liver and adipose tissue weight and adipocyte size. Serum lipid profiles and adiponectin levels were improved in HFD-fed mice treated with AFE but not AFW. However, both AFW and AFE significantly attenuated HFD-induced changes in serum leptin and insulin-like growth factor 1 levels, liver expression of AMPK, and adipose tissue expression of UCP2.

Conclusions: The findings from this study suggest that A. fistulosum extracts have potential as functional food materials for weight control in obesity.

Keywords: Adiponectin, Allium fistulosum, AMP-activated protein kinase

\section{Background}

Obesity is a major risk factor for dyslipidemia, type 2 diabetes, atherosclerosis, hypertension, cardiovascular disease, and certain cancers in the developed world $[1,2]$. Numerous treatments for obesity have been investigated, including the clinically available anti-obesity drug orlistat (Xenical), an intestinal lipase inhibitor [3]. However, orlistat can cause serious side effects such as insomnia, constipation, vomiting, emesis, headache, and stomachache [4]. Conjugated linoleic acid (CLA), which consists of a family of polyunsaturated fatty acids, is one of the most popular weight-loss

\footnotetext{
* Correspondence: hkkim@kiom.re.kr

${ }^{1}$ Mibyeong Research Center, Korea Institute of Oriental Medicine, 1672

Yuseong-daero, Yuseong-gu, Daejeon 305-811, Republic of Korea

Full list of author information is available at the end of the article
}

supplements in the world [5]. However, in large doses CLA can cause fat accumulation in the liver, which can lead to metabolic syndrome and diabetes $[6,7]$. Thus, there is a demand for alternative therapies, such as herbal medicines, with minimal side effects [8]. One popular weight-loss herbal supplement is Garcinia cambogia [9]. This edible fruit contains large quantities of hydroxycitric acid (HCA), which has fat mass loss and lipid-lowering effects [10]. G. cambogia protects against high-fat diet (HFD)-induced obesity by modulating adipose fatty acid synthesis and $\beta$ oxidation, but can induce hepatic fibrosis, inflammation, and oxidative stress [11].

Allium fistulosum (Welsh onion or bunching onion) is a species of perennial onion originated in Eastern Asia [12]. A. fistulosum is an important cooking ingredient in several 
Eastern countries, including China, Japan, and Korea [13]. In Western countries, $A$. fistulosum is used primarily as a scallion or salad onion, and is the species most commonly marketed for this purpose [14]. Its leaves have nutritional value and can be used fresh all year [15]. A. fistulosum also has traditional uses as an herbal medicine for the treatment for colds, influenza, abdominal pain, headache, constipation, dysentery, sores, ulcers, parasitic infestations, arthritis, and heart disease [16]. In addition, A. fistulosum has been shown to possess antifungal, antioxidative, antiplatelet, and antihypertensive properties [16-18]. Our previous studies showed that oral administration of $A$. fistulosum, either as a $70 \%$ ethanol extract or as a meal replacement cereal bar containing A. fistulosum ethanol extract, inhibited increases in body weight, adipose tissue mass, fat accumulation, and serum lipid levels in HFDinduced obese mice $[19,20]$. Nutritional component analysis showed that $A$. fistulosum extract powder contains low levels of total fat and is rich in vitamins (e.g., $\mathrm{B}_{2}, \mathrm{~B}_{6}$, niacin, and folic acid) and iron [20].

In the present preclinical study, the phytochemical contents of aqueous and ethanolic extracts of A. fistulosum were analyzed by high-performance liquid chromatography (HPLC). The effects of these extracts on body weight and other obesity-related parameters were explored in HFD-fed mice. The anti-obesity effects of these extracts were compared with the clinical weight-loss drug, orlistat, and the weight-loss supplements, G. cambogia extract containing HCA and CLA.

\section{Methods}

\section{Preparation of $A$. fistulosum extracts}

A. fistulosum was purchased as a dried herb from Omniherb Co. (Yeongcheon, Korea). A voucher specimen (No. PH-79 W and PH-79E) was deposited at the herbarium of the Department of Herbal Resources Research at the Korea Institute of Oriental Medicine. The dried bulbs and roots of $A$. fistulosum (100 g) were extracted twice with 10 volumes of water or $70 \%$ ethanol using a 2-h reflux extraction at $90{ }^{\circ} \mathrm{C}$. The extract was then concentrated under reduced pressure, filtered, lyophilized, and stored at $4{ }^{\circ} \mathrm{C}$. Yields of the aqueous and ethanolic extracts from the starting material were $11.25 \%$ and $15.91 \%$, respectively.

\section{HPLC determination of ferulic acid and quercetin}

The sample was analyzed by reverse-phase HPLC using a Waters Alliance 2695 system (Waters Co., Milford, MA, USA) coupled with a 2998 photodiode array detector. An INNO C18 column $(250 \mathrm{~mm} \times 4.6 \mathrm{~mm}$, particle size $5 \mu \mathrm{m}$; Young Jin Biochrom Co., Sungnam, Korea) was used as the stationary phase, and the mobile phase was composed of $0.1 \%(\mathrm{v} / \mathrm{v})$ trifluoroacetic aqueous solution (A) and acetonitrile (B). Elution conditions were as follows. At $t=$ 0 min the mobile phase consisted of $10 \% \mathrm{~A} / 90 \% \mathrm{~B}$ and was held for $10 \mathrm{~min}$. From 10 to $60 \mathrm{~min}$, a gradient was applied to $60 \% \mathrm{~A} / 40 \% \mathrm{~B}$, which was followed by a wash with $100 \%$ B for 5 min and a 15-min equilibration period at $90 \% \mathrm{~A} / 10 \% \mathrm{~B}$. The separation temperature was kept constant at $40{ }^{\circ} \mathrm{C}$ throughout the analysis, with a flow rate of $1.0 \mathrm{~mL} / \mathrm{min}$ and injection volume of $20 \mu \mathrm{L}$. Identification was based on retention time and UV spectra using comparison with commercial standards. The components were quantified based on peak areas at the maximal wavelength. Calibration curves of the standards, ranging from 3.125 to $100 \mu \mathrm{g} / \mathrm{mL}$ (six levels), showed good linearity, with $R^{2}$ values exceeding 0.99 (peak areas vs. concentration). HPLC-grade reagents, acetonitrile, and water were obtained from J. T. Baker (Phillipsburg, NJ, USA). The standards of ferulic acid and quercetin (purity > 98\%) were purchased from Sigma-Aldrich Co. (St. Louis, MO, USA).

\section{Animals and experimental diets}

Male 8-week-old C57BL/6 J mice were purchased from The Jackson Laboratory (Bar Harbor, ME, USA). The mice were housed in an air-conditioned animal room with a 12h light/12-h dark cycle at a temperature of $21 \pm 2{ }^{\circ} \mathrm{C}$ and humidity of $50 \pm 5 \%$. The mice were fed a commercial diet (Ralston Purina, St. Louis, MO, USA) for 1 week, with food and water provided ad libitum.

The mice were then randomly divided into the following seven groups ( $n=6$ each) according to treatment: standard chow diet (normal control), HFD alone (HFD-control), HFD plus G. cambogia extract containing HCA (HFD-HCA), HFD plus conjugated linoleic acid (HFDCLA), HFD plus orlistat (HFD-ORL), HFD plus A. fistulosum aqueous extract (HFD-AFW), or HFD plus A. fistulosum ethanolic extract (HFD-AFE). The mice receiving HCA, CLA, or ORL served as positive controls. The standard chow (Orient Bio Inc., Seongnam, Korea) provided $14 \%$ of energy as fat, $21 \%$ as protein, and $65 \%$ as carbohydrates. The HFD (Rodent Diet D12492, Research Diet, New Brunswick, NJ) provided 60\% of energy as fat, $20 \%$ as protein, and $20 \%$ as carbohydrates. HCA, CLA, AFW, and AFE were dissolved in normal saline and orally administrated at a dose of $100 \mathrm{mg} / \mathrm{kg} /$ day. Orlistat (Xenical, Roche Korea Co., Seoul, Korea) was orally administered at a dose of $15.6 \mathrm{mg} / \mathrm{kg}$, which was based on the human dosage. The normal control and HFD-control groups received vehicle (normal saline) only. Body weight and food intake were determined every week during the 6-week experimental period. The food efficiency ratio was calculated as the daily body weight gain divided by the daily food intake [21].

This study adhered to the Guide for the Care and Use of Laboratory Animals developed by the Institute of Laboratory Animal Resources of the National Research Council and was approved by the Institutional Animal Care and 
Table 1 The primer and probe sequences used in the real-time RT-PCR analysis

\begin{tabular}{lll}
\hline Genes & $\begin{array}{l}\text { Probe and } \\
\text { Primer }\end{array}$ & Sequence \\
\hline Leptin & sense & 5'-AACCCTAACTGAACTCAGATTGTTAG-3' \\
& antisense & 5'-TAAGTCAGTTAAATGCTTAGGG-3' \\
UCP-2 & sense & 5'-TTCAAATGAGATTGTGGGAAAAT-3' \\
& antisense & 5'-ACCGATACAGTACAGTACAGTA-3' \\
Adiponectin & sense & 5'-CCCAAGGGAACTTGTGCAGGTTGGATG-3' \\
& antisense & 5'-GTTGGTATCATGGTAGAGAAGAAAGCC-3' \\
AMPKa2 & sense & 5'-GATGATGAGGTGGTGGA-3' \\
& antisense & 5'-GCCGAGGACAAAGTGC-3' $^{\prime}$ \\
AMPKa1 & sense & 5'-AAGCCGACCCAATGACATCA-3' $^{\prime}$ \\
& antisense & 5'-CTTCCTTCGTACACGCAAAT-3' \\
PPAR- $y$ & FAM & 5'-TCGGAATCAGCTCTGTGGACCTCTCC-3' \\
GAPDH & VIC & 5'-TGCATCCTGCACCACCAACTGCTTAG-3' \\
\hline
\end{tabular}

Use Committee of Daejeon University in Daejeon, Korea (Permit No. DJUARB2014-042).

\section{Serum biochemistry}

At the end of the 6 weeks, the mice were anesthetized with pentobarbital (intraperitoneally, $100 \mathrm{mg} / \mathrm{kg}$ ) after an overnight fasting. Blood samples were collected from the abdominal aorta into a vacuum collection tube (BD Biosciences, San Diego, CA, USA). Total cholesterol, high-density lipoprotein (HDL)-cholesterol, low-density lipoprotein (LDL)-cholesterol, triacylglycerol, free fatty acids, glucose, creatinine, aspartate transaminase (AST), and alanine transaminase (ALT) concentrations were determined using an Express Plus automatic analyzer (Chiron Diagnostics, East Walpole, MA, USA). Serum leptin, adiponectin, and insulin-like growth factor I (IGF-1) levels were determined using commercially available mouse enzyme-linked immunosorbent assay kits
(Diagnostic Systems Laboratories, Inc., Webster, TX, USA and Linco Research, St Charles, MO, USA) [22].

\section{Histologic analysis of liver and adipose tissues}

After collecting the blood, white adipose tissues (subcutaneous, epididymal, and perirenal fat) [23] and livers were removed from the mice and weighed immediately. Liver and epididymal adipose tissues were fixed in 10\% neutral buffered formalin for 1 day and then embedded in paraffin. The tissues were cut $(6-\mu \mathrm{m}$ thickness) and stained with hematoxylin and eosin. Adipocyte size was determined in the stained sections using light microscopy (Olympus BX51, Olympus Optical Co., Tokyo, Japan) and an image analysis program (Image-Pro Plus 5.0, Media Cybernetics, Silver Spring, MD, USA).

Real-time reverse-transcription polymerase chain reaction Total RNA from liver and epididymal adipose tissue was isolated with TRI Reagent (Sigma-Aldrich) and then digested with DNase I (Life Technologies, Grand Island, NY, USA) to remove chromosomal DNA (cDNA). Total RNA $(\mu \mathrm{g})$ was reverse transcribed into cDNA with the First Strand cDNA Synthesis Kit (Amersham Pharmacia, Piscataway, NJ, USA). Real-time reverse-transcription polymerase chain reaction (RT-PCR) was performed using the Applied Biosystems 7500 Real-Time PCR system (Applied Biosystems). The primer and probe sequences are shown in Table 1. The probes were labeled with 6carboxyfluorescein, a fluorescent reporter dye. PCR reactions were carried out with TaqMan Universal PCR Master Mix containing DNA polymerase (Applied Biosystems) according to the manufacturer's instructions, using the following PCR conditions: $2 \mathrm{~min}$ at $50{ }^{\circ} \mathrm{C}, 10 \mathrm{~min}$ at $95{ }^{\circ} \mathrm{C}$, followed by 40 cycles of $15 \mathrm{~s}$ at $95^{\circ} \mathrm{C}$ and $1 \mathrm{~min}$ at $60{ }^{\circ} \mathrm{C}$. Relative target gene expression was determined using the comparative $\mathrm{Ct}$ (threshold cycle number at the cross-point between the amplification plot and threshold) method with
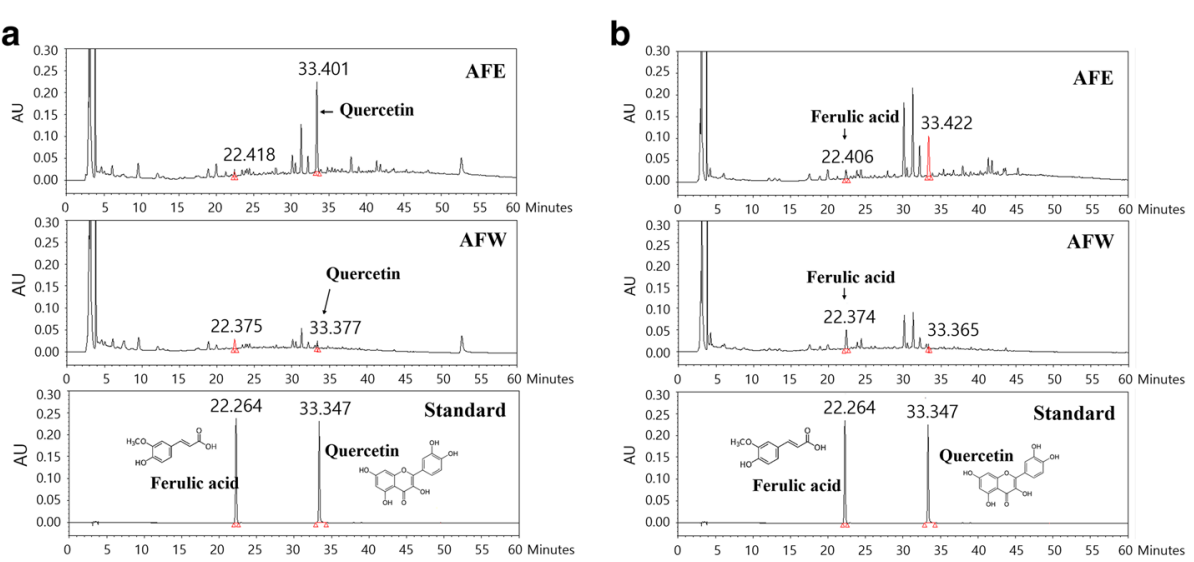

Fig. 1 High-performance liquid chromatography/photodiode array detector chromatograms of Allium fistulosum aqueous extract (AFW), A. fistulosum ethanolic extract (AFE), and standards for quercetin and ferulic acid. Quercetin and ferulic acid were detected at (a) $254 \mathrm{~nm}$ and (b) $300 \mathrm{~nm}$, respectively 
glyceraldehyde 3-phosphate dehydrogenase as an internal control. The expression levels of mRNA were normalized to those of GAPDH and calculated using the $2^{-\Delta \Delta \mathrm{Ct}}$ method according to the manufacturer's instructions.

\section{Statistical analysis}

Groups were compared by one-way analysis of variance followed by Duncan's multiple range post hoc test. Results are expressed as the mean \pm standard error of the mean (SEM); $p<0.05$ was considered significant.

\section{Results}

\section{HPLC analysis}

Ferulic acid and quercetin were identified in A. fistulosum extracts by HPLC-photodiode array detection. As shown in Fig. 1, the retention times of ferulic acid and quercetin were approximately $22 \mathrm{~min}$ and $33 \mathrm{~min}$, respectively. AFE contained $0.17 \pm 0.02 \mathrm{mg} / \mathrm{g}$ ferulic acid and $2.22 \pm$ $0.01 \mathrm{mg} / \mathrm{g}$ quercetin, and AFW contained $0.38 \pm 0.02 \mathrm{mg} /$ $\mathrm{g}$ ferulic acid and $0.43 \pm 0.01 \mathrm{mg} / \mathrm{g}$ quercetin (Table 2).

\section{Changes in body weight, food intake, and food efficiency ratio}

Body weight, body weight gain, food intake, and food efficiency ratios of mice fed standard chow (normal control),
Table 2 Content of components in the A. fistulosum extracts

\begin{tabular}{lll}
\hline & AFW $(\mathrm{mg} / \mathrm{g})$ & AFE $(\mathrm{mg} / \mathrm{g})$ \\
\hline Ferulic acid & $0.38 \pm 0.02$ & $0.17 \pm 0.02$ \\
Quercetin & $0.43 \pm 0.01$ & $2.22 \pm 0.01$
\end{tabular}

HFD-control, or HFD plus HCA, CLA, ORL, AFW or AFE, for 6 weeks are shown in Fig. 1. Our results showed that the final body weight of HFD-fed mice was approximately $31.4 \%$ higher than that of normal controls (Fig. 2a). However, the final body weights of HFD-fed mice that also received HCA, CLA, or ORL (positive controls) were significantly decreased $(p<0.05)$ by approximately $11.9 \%$, $17.4 \%$, and $21.2 \%$, respectively, compared with the HFDcontrol group. Similarly, the final body weights of HFDfed mice that also received AFW or AFE were decreased by $14.8 \%$ and $15.0 \%$, respectively, compared with the HFD-control group. Body weight gain also was significantly decreased in the AFW- and AFE-treated mice and in the HCA-, CLA-, and ORL-treated controls compared with the HFD-control group (Fig. 2b), although food intake did not differ significantly between the groups (Fig. 2c). The food efficiency ratio of HFD-fed mice was 4.12 times that of normal controls but was significantly decreased in HFD-fed mice that also received CLA, ORL, or a
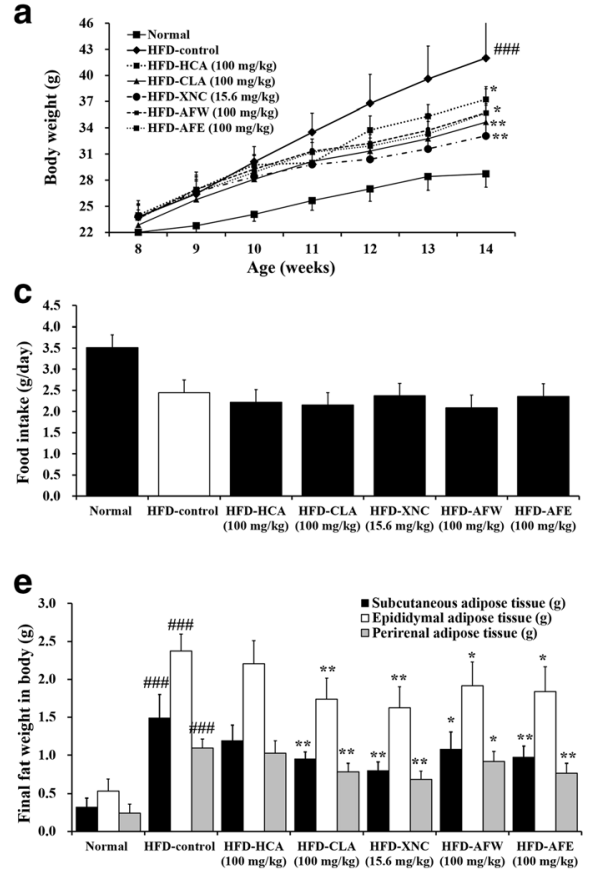

b

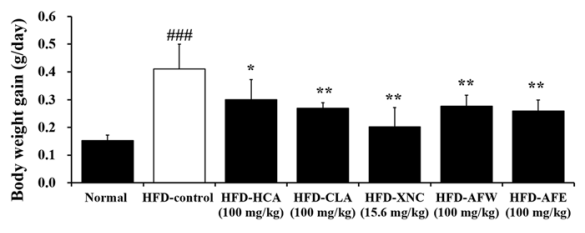

d

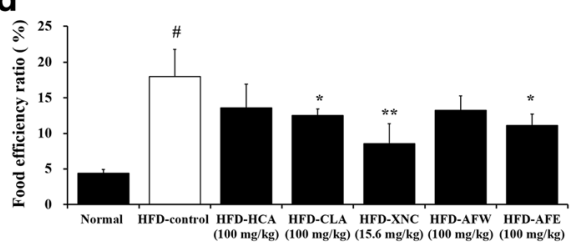

f

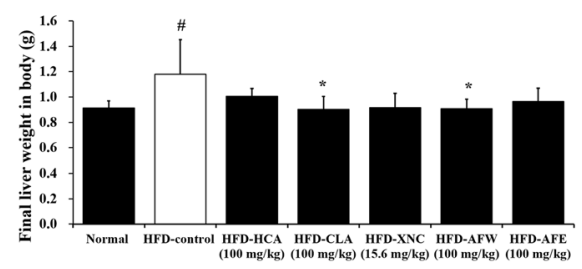

Fig. 2 Effect of 6-week treatment with Allium fistulosum aqueous extract (AFW) and A. fistulosum ethanolic extract (AFE) in high-fat diet (HFD)-fed mice. a Body weight, (b) body weight gain, (c) food intake, (d) food efficiency ratio, (e) final adipose tissue weight, and (f) final liver weight. Normal, standard chow; HFD-control, HFD only; HFD-HCA, HFD plus 100 mg/kg Garcinia cambogia extract containing hydroxycitric acid; HFD-CLA, HFD plus 100 mg/kg conjugated linoleic acid; HFD-ORL, HFD plus 15.6 mg/kg Xenical (orlistat); HFD-AFW, HFD plus 100 mg/kg AFW; HFD-AFE, HFD plus $100 \mathrm{mg} / \mathrm{kg}$ AFE. Results are expressed as the mean \pm standard error of the mean (SEM) $(n=6)$. \# $p<0.05$, \#\#\# $p<0.001$ compared with the normal control group; ${ }^{*} p<0.05,{ }^{* *} p<0.01$ compared with the HFD-control group 
AFE (38.3\% lower) (Fig. 2d). The food efficiency ratio appeared to be $26.5 \%$ lower in AFW-treated mice compared with the HFD-control group, but this difference was not significant. These results suggest that AFW and AFE can inhibit HFD-induced body weight gain.

\section{Adipose tissue and liver weights}

We found that the weights of subcutaneous, epididymal, and perirenal adipose tissues in the HFD-fed mice were $4.6,4.5$, and 4.6 times that of the normal controls, respectively. However, this HFD-induced increase in adipose tissue weight was significantly decreased by treatment with CLA, ORL, AFW, and AFE (Fig. 2e). The liver weight of HFD-fed mice was 1.3 times that of normal control mice, but this increase was significantly attenuated by treatment with CLA and AFW (Fig. 2f).

\section{Measurement of serum lipid and glucose levels}

The effects of $A$. fistulosum extracts on serum lipid levels and glucose are shown in Fig. 3a-f. Our results show that triacylglycerol, total cholesterol, LDLcholesterol, and free fatty acid levels were higher in HFD-fed mice compared with normal controls. Treatment with HCA, CLA, ORL, AFW, and AFE significantly attenuated the HFD-induced increase in triacylglycerol and free fatty acid concentrations $(p<$ $0.05)$. In addition, the HFD-induced increases in total cholesterol and LDL-cholesterol levels were significantly attenuated in CLA-, ORL-, and AFE-treated mice. HDL-cholesterol level was significantly elevated in the treatment groups compared with the HFDcontrol group and normal controls. Glucose levels of HFD-fed mice were higher than those of the normal controls but were not significantly decreased in the mice treated with HCA, CLA, ORL, AFW, or AFE.
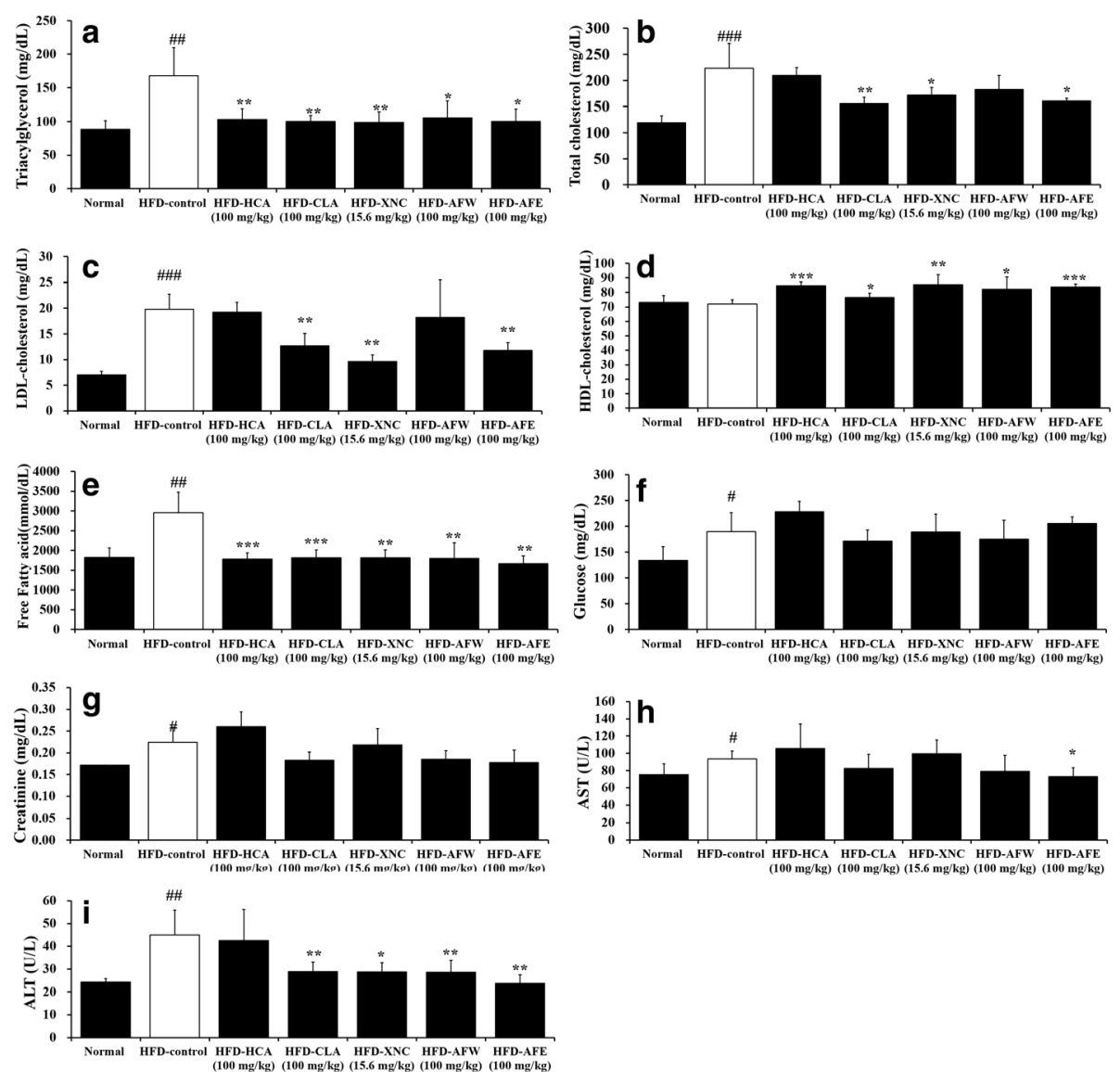

Fig. 3 Effect of 6-week treatment with Allium fistulosum aqueous extract (AFW) and A. fistulosum ethanolic extract (AFE) on blood lipid and glucose levels and liver enzymes in high-fat diet (HFD)-induced obese mice. a Triacylglycerol, (b) total cholesterol, (c) LDL-cholesterol, (d) HDL-cholesterol, (e) free fatty acids, (f) glucose, (g) creatinine, (h) aspartate transaminase (AST), and (i) alanine transaminase (ALT) levels. Normal, standard chow; HFDcontrol, HFD only; HFD-HCA, HFD plus 100 mg/kg Garcinia cambogia extract containing hydroxycitric acid; HFD-CLA, HFD plus 100 mg/kg conjugated linoleic acid; HFD-ORL, HFD plus 15.6 mg/kg Xenical (orlistat); HFD-AFW, HFD plus 100 mg/kg AFW; HFD-AFE, HFD plus 100 mg/kg AFE. Results are expressed as the mean \pm standard error of the mean (SEM) $(n=6)$. \# $p<0.05$, \#\# $p<0.01$, \#\#\# $p<0.001$ compared with the normal control group; * $p$ $<0.05,{ }^{* *} p<0.01,{ }^{* * *} p<0.001$ compared with the HFD-control group 


\section{Assessment of liver and kidney function}

To evaluate any potential toxic effects of AFW and AFE, liver and kidney function were evaluated at the end of the 6-week experimental period (Fig. 3g-i). The level of blood creatinine, an indicator of kidney function, was increased in HFD-fed mice, but was significantly attenuated in mice treated with CLA, AFW, and AFE. AST and ALT, two indicators of liver function, also were increased in HFD-fed mice compared with normal controls. The HFDinduced increase in ALT was significantly attenuated in mice treated with CLA, ORL, AFW, and AFE; however, the HFD-induced increase in AST was significantly attenuated only in mice treated with AFE.

\section{Serum adipocytokine and IGF-1 concentrations}

HFD-fed mice exhibited significantly elevated serum leptin levels compared with normal controls. This increase was attenuated by treatment with CLA, ORL, AFW, and AFE but not with HCA (Fig. 4a). Serum adiponectin levels were not significantly different in HFD-fed mice compared with controls but were increased in mice treated with HCA and AFE (Fig. 4b). Serum IGF-1 levels were significantly decreased in all treated experimental groups compared with the HFD-control group (Fig. 4c).

\section{Histopathological examination and gene expression in liver}

Histologic examination of the liver showed numerous lipid droplets in the HFD-fed mice but not in the normal controls (Fig. 5a). This HFD-induced lipid accumulation was attenuated in mice that received CLA, ORL, AFW, and AFE. To identify the molecular mechanism underlying the effect of AFW and AFE on lipid accumulation in the liver, we evaluated the expression of major lipid metabolism-related genes in the liver by real-time RTPCR. Mice treated with ORL, AFW, and AFE showed significantly increased mRNA levels of the adenosine monophosphate-activated protein kinase (AMPK) isoforms $A M P K \alpha 1$ and $A M P K \alpha 2$ compared with normal controls and HFD-control mice (Fig. 5b).

\section{Adipocyte size and gene expression in epididymal adipose tissue}

Examination of epididymal adipose tissue sections showed significantly increased adipocyte size in the HFD-control mice compared with normal controls (Fig. 6a). The increase in adipocyte area was attenuated by HCA, CLA, ORL, AFW, and AFE (Fig. 6b). Results of real-time RT-PCR showed that mRNA levels of $A M P K \alpha 1$ and $A M P K \alpha 2$ in epididymal adipose tissue were not significantly higher in HFD-control mice compared with normal controls (Fig. 7a and b). Leptin mRNA levels were significantly higher in HFD-control mice compared with normal controls. This increase was

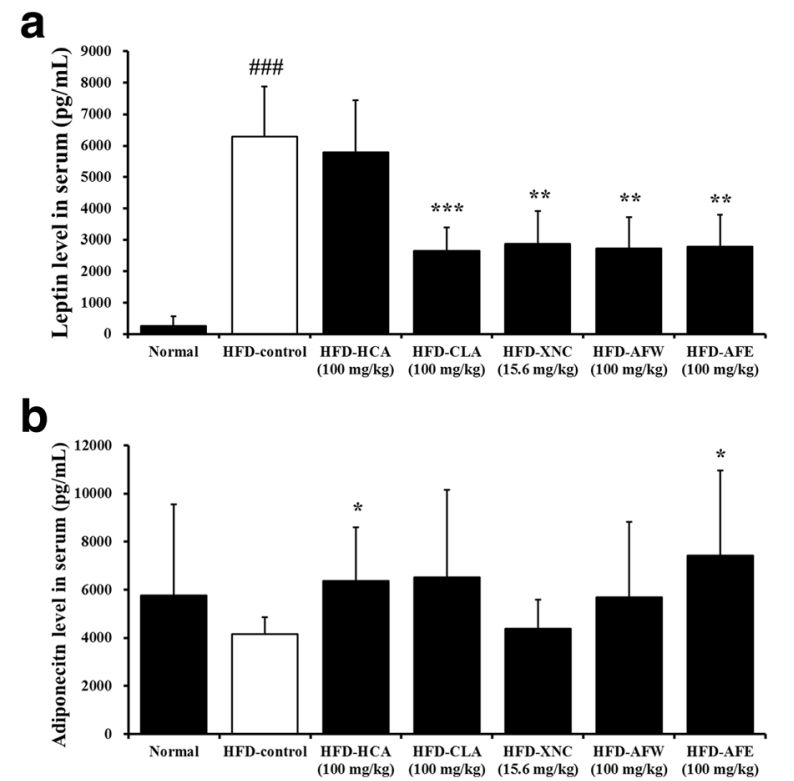

Fig. 4 Effect of 6-week treatment with Allium fistulosum aqueous extract (AFW) and A. fistulosum ethanolic extract (AFE) on serum adipocytokine and IGF-1 concentrations in high-fat diet (HFD)-induced obese mice, as assessed by enzyme-linked immunosorbent assay. Serum levels of (a) leptin, (b) adiponectin, and (c) IGF-1. Results are expressed as the mean \pm standard error of the mean (SEM) $(n=6)$. Normal, standard chow; HFD-control, HFD only; HFDHCA, HFD plus 100 mg/kg Garcinia cambogia extract containing hydroxycitric acid; HFD-CLA, HFD plus 100 mg/kg conjugated linoleic acid; HFD-ORL, HFD plus 15.6 mg/kg Xenical (orlistat); HFD-AFW, HFD plus 100 mg/kg AFW; HFD-AFE, HFD plus 100 mg/kg AFE. \#\#\# $p<0.001$ compared with the normal control group; ${ }^{*} p<0.05$, ${ }^{* *} p$ $<0.01$, *** $p<0.001$ compared with the HFD-control group

not significantly attenuated by treatment with $\mathrm{HCA}$, CLA, ORL, AFW, and AFE (Fig. 7c). In contrast, adiponectin mRNA levels were significantly higher in all treatment groups compared with normal controls and HFDfed controls (Fig. 7d). Uncoupling protein 2 (UCP2) mRNA levels were significantly lower in HFD-fed mice compared with normal controls, but the HFD-induced decrease was significantly attenuated by all treatments (Fig. 7e). Peroxisome proliferator-activated receptor gamma (PPAR- $\gamma$ ) mRNA levels were decreased in mice receiving CLA, ORL, and AFE compared with normal controls and mice receiving the HFD only (Fig. 7f).

\section{Discussion}

In the present study, oral administration of AFW and AFE attenuated the HFD-induced increase in serum levels of triacylglycerol, free fatty acids, and leptin; lipid accumulation in the liver; liver and adipose tissue weight; adipocyte size; and body weight in mice. In addition, AFE attenuated the HFD-induced increase in total cholesterol and LDLcholesterol. We found that serum HDL-cholesterol and adiponectin levels were higher in HFD-fed mice treated 

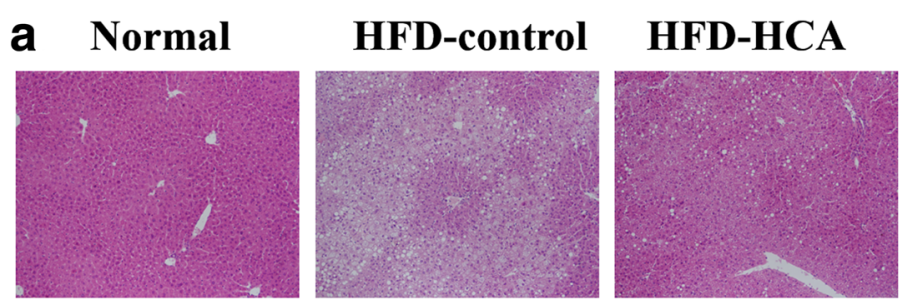

HFD-CLA

\section{HFD-XNC}

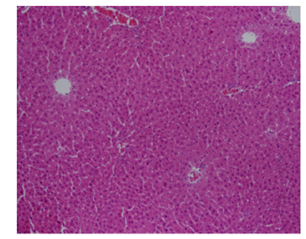

HFD-AFW

\section{HFD-AFE}
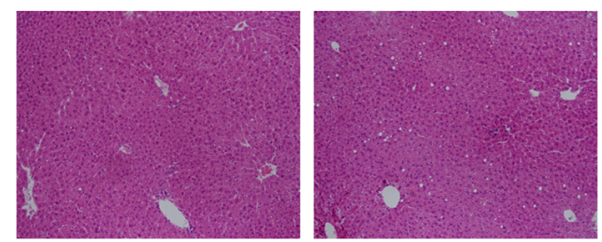

b

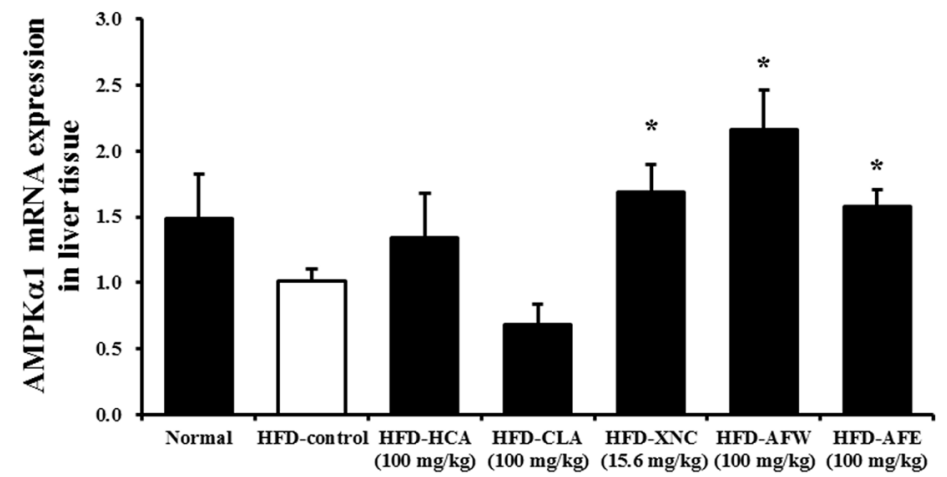

C

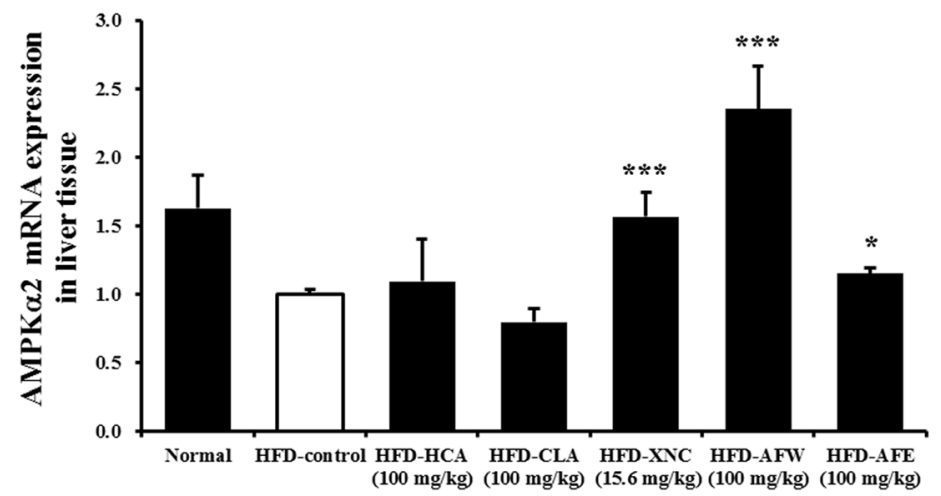

Fig. 5 Effect of 6-week treatment with Allium fistulosum aqueous extract (AFW) and A. fistulosum ethanolic extract (AFE) on histology and gene expression in the liver tissue of high-fat diet (HFD)-induced obese mice. a Representative photographs of hematoxylin and eosin-stained liver sections (magnification, 400X). b Expression of adenosine monophosphate-activated protein kinase (AMPK) isoforms AMPKa1 and AMPKa2 mRNA in the liver, as assessed by realtime reverse-transcription polymerase chain reaction (RT-PCR). Normal, standard chow; HFD-control, HFD only; HFD-HCA, HFD plus 100 mg/kg Garcinia cambogia extract containing hydroxycitric acid; HFD-CLA, HFD plus 100 mg/kg conjugated linoleic acid; HFD-ORL, HFD plus 15.6 mg/kg Xenical (orlistat); HFD-AFW, HFD plus $100 \mathrm{mg} / \mathrm{kg}$ AFW; HFD-AFE, HFD plus $100 \mathrm{mg} / \mathrm{kg}$ AFE. Results are expressed as the mean \pm standard error of the mean (SEM) $(n=6)$. ${ }^{*}$ $p<0.05,{ }^{* * *} p<0.001$ compared with the HFD-control group

with AFW and AFE than in normal controls and mice receiving the HFD only.

The insulin/IGF-1 axis plays an important role in the association between obesity and risk of breast cancer [24]. Some studies have demonstrated that obese children exhibit the highest levels of serum
IGF-1 and also exhibit a positive relationship between IGF-1 and adiposity, which perhaps contributes to their increased risk of obesity-related cancers [25]. In this study, the HFD-induced increase in serum IGF-1 levels was significantly attenuated in mice treated with AFW and AFE. Expression of AMPK, which is a 


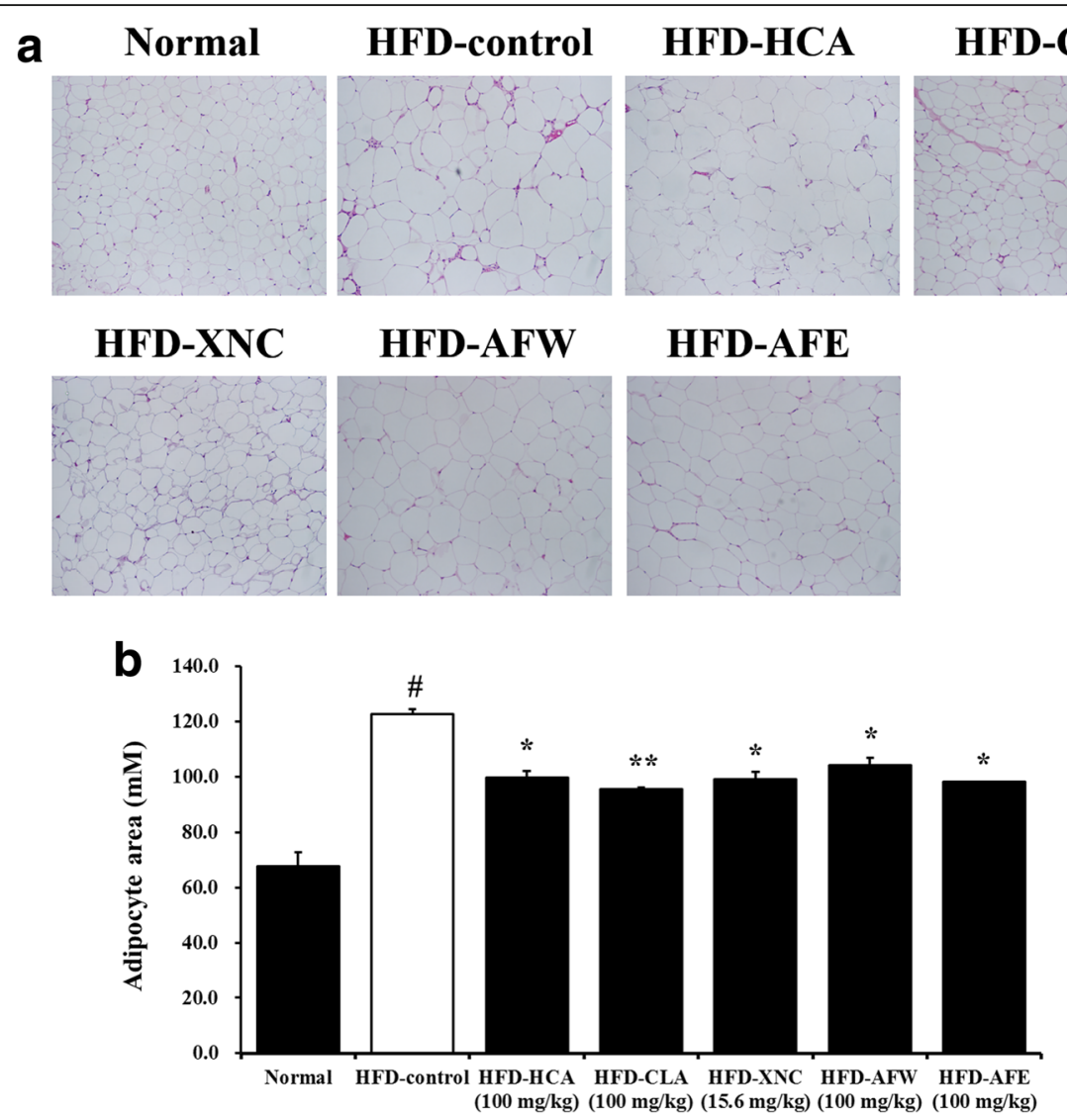

Fig. 6 Effect of 6-week treatment with Allium fistulosum aqueous extract (AFW) and A. fistulosum ethanolic extract (AFE) on adipocyte size in epididymal adipose tissue of high-fat diet (HFD)-induced obese mice. a Representative photographs of adipose tissue of hematoxylin and eosin-stained tissue sections (magnification, 400x). b Adipocyte area of hematoxylin and eosin-stained adipose tissue sections. Normal, standard chow; HFD-control, HFD only; HFD-HCA, HFD plus 100 mg/kg Garcinia cambogia extract containing hydroxycitric acid; HFD-CLA, HFD plus 100 mg/kg conjugated linoleic acid; HFD-ORL, HFD plus 15.6 mg/kg Xenical (orlistat); HFD-AFW, HFD plus 100 mg/kg AFW; HFD-AFE, HFD plus 100 mg/kg AFE. Results are expressed as the mean \pm standard error of the mean (SEM) $(n=6)$. \# $p<0.05$ compared with the normal control group; ${ }^{*} p<0.05$, ** $p<0.01$ compared with the HFD-control group

cellular energy sensor involved in energy homeostasis, was evaluated in adipose tissue and liver. AMPK triggers catabolic pathways, such as glucose transport and fatty acid $\beta$-oxidation, and inhibits anabolic pathways such as fatty acid, cholesterol, and protein synthesis [26]. Because of its critical role in metabolism, AMPK is a target for drugs used to treat cancer, diabetes, and metabolic syndrome [27]. Our results show that treating HFD-fed mice with AFW or AFE increased mRNA levels of AMPK $\alpha$ in the liver, suggesting that AFW and AFE may lower lipid accumulation and serum lipid levels through the regulation of lipid metabolism in the liver. In adipose tissue, AFW and AFE increased UCP2 and adiponectin expression. In addition, AFE decreased the expression of PPAR- $\gamma$, which is a ligand-activated transcription factor that plays an important role in adipocyte differentiation and lipid storage [28] and regulates the expression of adiponectin through a PPAR-responsive element in its promoter [29]. UCP2, a mitochondria inner membrane transporter, promotes fatty acid oxidation in adipose tissue and lowers body weight [30].

Adiponectin is a hormone secreted by adipose tissue that modulates glucose and lipid metabolism by stimulating fatty acid oxidation in almost all its major target tissues, including skeletal muscle, liver, and adipocytes [31]. The beneficial effects of adiponectin in these target tissues are dramatically attenuated in UCP2-deficient mice and by expression of dominant negative AMPK [32]. In patients with obesity and diabetes, plasma adiponectin levels are inversely correlated with body fat content, suggesting that adiponectin is an important factor for the treatment of obesity [33, 34]. In the present study, it was found that serum adiponectin levels and adiponectin mRNA levels in adipose tissue were higher in AFE-treated mice compared with mice receiving the HFD only. Conversely, the HFD-induced increase in serum leptin levels was significantly attenuated in mice 

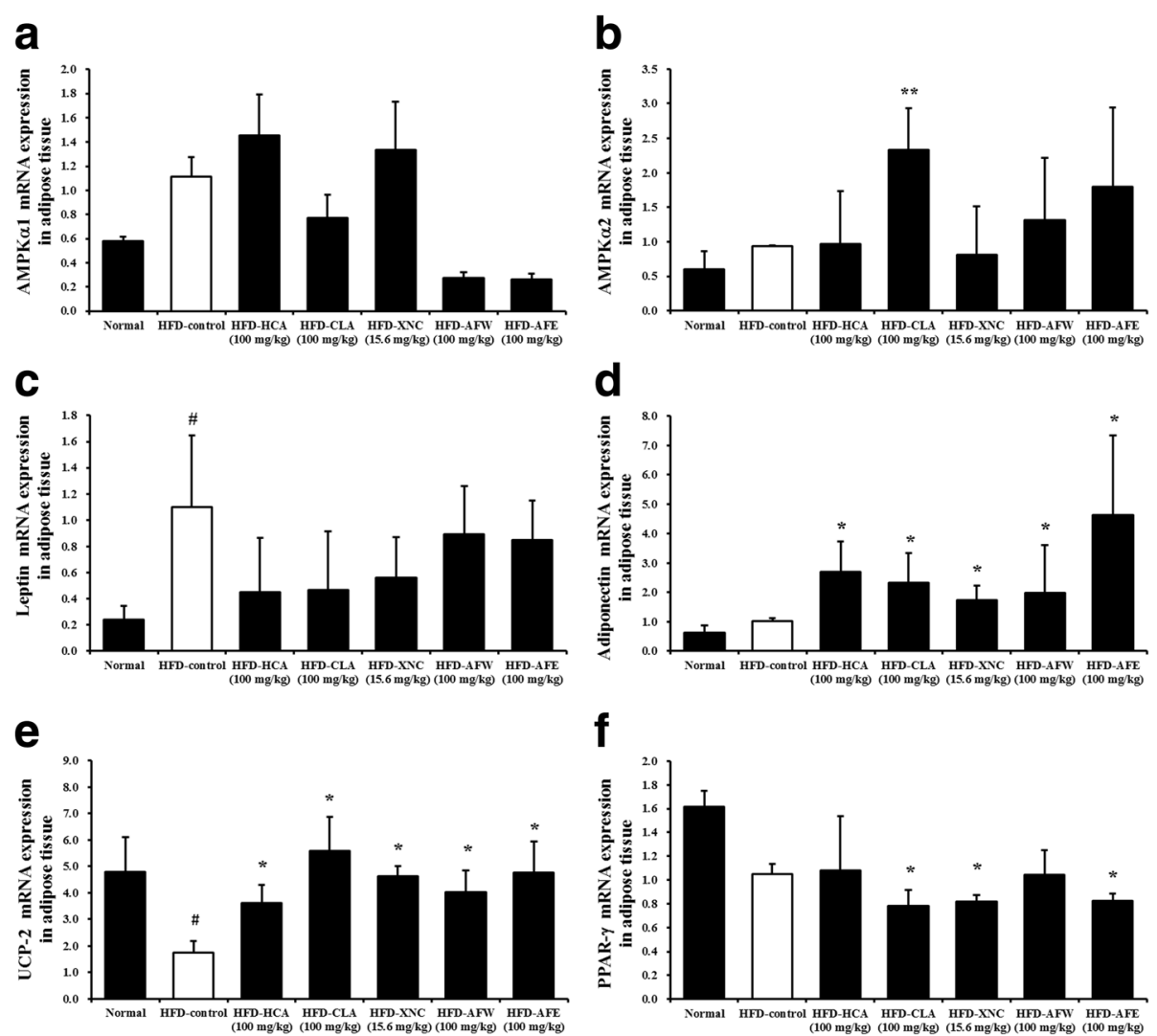

Fig. 7 Effect of 6-week treatment with Allium fistulosum aqueous extract (AFW) and A. fistulosum ethanolic extract (AFE) on gene expression in epididymal adipose tissue of high-fat diet (HFD)-induced obese mice. Expression levels of (a) adenosine monophosphate-activated protein kinase (AMPK) isoforms AMPKa1, (b) AMPKa2, (c) leptin, (d) adiponectin, (e) uncoupling protein 2 (UCP2), and (f) peroxisome proliferator-activated receptor gamma (PPAR-ү), as assessed by real-time reverse-transcription polymerase chain reaction (RT-PCR). Normal, standard chow; HFD-control, HFD only; HFD-HCA, HFD plus 100 mg/kg Garcinia cambogia extract containing hydroxycitric acid; HFD-CLA, HFD plus 100 mg/kg conjugated linoleic acid; HFD-ORL, HFD plus 15.6 mg/ $\mathrm{kg}$ Xenical (orlistat); HFD-AFW, HFD plus $100 \mathrm{mg} / \mathrm{kg}$ AFW; HFD-AFE, HFD plus $100 \mathrm{mg} / \mathrm{kg}$ AFE. Results are expressed as the mean \pm standard error of the mean (SEM) $(n=6)$. \# $p<0.05$ compared with the normal control group; ${ }^{*} p<0.05,{ }^{* *} p<0.01$ compared with the HFD-control group

treated with AFW and AFE, although leptin mRNA levels in adipose tissue were not significantly decreased in the treatment groups. Blood levels of leptin are positively associated with adiposity and increased body weight in humans and rodents [35]. These results suggest that the regulation of adiponectin and lipid metabolism-related genes expression by AFW and AFE may be responsible for the decrease in serum lipid levels and the attenuation of adiposity in HFD mice.

HPLC analysis identified ferulic acid and quercetin in AFE and AFW. Ethanol and water are the most common solvent to extract the bioactive compounds of herbal medicine for HPLC analysis and traditional decoction, respectively. Our HPLC analysis identified ferulic acid and quercetin in AFE and AFW. The ferulic acid concentration in AFW was 2 times that of AFE and the quercetin concentration in AFE was 5.2 times that of AFW. The HPLC analysis was performed using commercial standards and compound libraries. Other compounds containing vanillic acid, tyrosol, 4-hydroxybenzaldehyde, and koumine were detected in the small peaks, but the content of these compounds in AFE and AFW was extremely low. Quercetin and ferulic acid, which are naturally occurring polyphenolic flavonoid compounds in fruits and vegetables, have been reported to suppress body weight, fat accumulation and hyperlipidemia in obese mice by enhancing antioxidant activities [36, 37]. The anti-obesity effect of quercetin is mediated by the AMPK signaling pathway in 3 T3-L1 adipocytes [38]. Structurally, hydroxyl groups of these flavonoids participate in radical stabilization via electron delocalization, thus contributing to the strong antioxidant and anti-obesity activities [39-41]. These phenolic compounds therefore may be partly responsible for the anti-obesity effects of AFW and AFE. In this study, especially, the quercetin concentration in AFE was high. Among the flavonoids, quercetin is an effective protector against lipid oxidation due to the presence and location of five hydroxyl groups on the aromatic ring [42]. Additionally, AFE exerted the stronger anti-obesity effects than AFW by enhancing adiponectin levels and reducing serum cholesterol levels in obese mice. 
Thus, this difference in anti-obesity action might be attributed to their chemical difference of quercetin.

However, it cannot be excluded that other components of these extracts may contribute to their anti-obesity activities. It has been reported that A. fistulosum contains $p$-coumaric acid, isoquercitrin, quercitrin, stigmasterol, $\beta$-sitosterol, campesterol, and allicin $[15,43]$. Thus, further study is needed to determine the anti-obesity components of A. fistulosum, including the development of optimized methods of chemical analysis to identify the bioactive compounds in the AFE and AFW extracts.

\section{Conclusions}

This study showed that the oral administration of AFW and AFE significantly reduced body weight, adipose tissue and liver weights, and fat accumulation in a mouse model of obesity. In addition, AFW and AFE improved the serum levels of triacylglycerol, total cholesterol, LDL-cholesterol, free fatty acids, and HDL-cholesterol. These findings indicate that $A$. fistulosum may be useful as a functional food material or therapeutic agent for the treatment of obesity and obesity-associated metabolic syndrome.

\section{Abbreviations}

AFE: A. fistulosum ethanolic extract; AFW: A. fistulosum aqueous extract; ALT: Alanine aminotransferase; AMPK: AMP-activated protein kinase; AST: Aspartate aminotransferase; CLA: Conjugated linoleic acid; HCA: Garcinia cambogia extract containing hydroxycitric acid; HDL: High-density lipoprotein; HFD: High-fat diet; HPLC: High-performance liquid chromatography; IGF: Insulin-like growth factor; ORL: Orlistat; PPAR$\gamma$ : Peroxisome proliferator-activated receptor- $\gamma ;$ RT-PCR: Reverse-transcription polymerase chain reaction; SEM: Standard error of the mean;

UCP2: Uncoupling protein 2

\section{Acknowledgments}

Not applicable.

\section{Funding}

This research was supported by the research program of the Korea Institute of Oriental Medicine, South Korea (K17091).

\section{Availability of data and materials}

The datasets used and/or analyzed during the current study available from the corresponding author on reasonable request.

\section{Authors' contributions}

YYS, DSK, and HYK participated in the design of the study. YYS carried out the experiments, analyzed the data, and wrote the manuscript. DSK carried out the HPLC analysis. SHK carried out the animal studies. All authors read and approved the final manuscript.

\section{Ethics approval and consent to participate}

All animal studies were approved by the Committee on Animal Care of the Daejeon University (Permit No. DJUARB2014-042).

\section{Consent for publication}

\section{Not applicable.}

\section{Competing interests}

The authors declare that they have no competing interests.

\section{Publisher's Note}

Springer Nature remains neutral with regard to jurisdictional claims in published maps and institutional affiliations.

\section{Author details}

${ }^{1}$ Mibyeong Research Center, Korea Institute of Oriental Medicine, 1672 Yuseong-daero, Yuseong-gu, Daejeon 305-811, Republic of Korea. ${ }^{2} \mathrm{KM}$ Convergence Research Division, Korea Institute of Oriental Medicine, Daejeon 305-811, Republic of Korea. ${ }^{3}$ Institute of Traditional Medicine and Bioscience, Daejeon University, Daejeon 300-716, Republic of Korea.

Received: 26 June 2017 Accepted: 1 March 2018

Published online: 20 March 2018

\section{References}

1. Evans RM, Barish GD, Wang YX. PPARs and the complex journey to obesity. Nat Med. 2004;10:355-61.

2. Watanabe T, Hata K, Hiwatashi K, Hori K, Suzuki N, Itoh H. Suppression of murine preadipocyte differentiation and reduction of visceral fat accumulation by a Petasites japonicus ethanol extract in mice fed a high-fat diet. Biosci Biotechnol Biochem. 2010;74:499-503.

3. Sung YY, Kim DS, Kim HK. Viola mandshurica ethanolic extract prevents high-fat-diet-induced obesity in mice by activating AMP-activated protein kinase. Environ Toxicol Pharmacol. 2014;38:41-50.

4. Bray GA. Medical therapy for obesity. Mt Sinai J Med. 2010;77:407-17.

5. Gudbrandsen OA, Rodríguez E, Wergedahl H, Mørk S, Reseland JE, Skorve J, Palou A, Berge RK. Trans-10, cis-12-conjugated linoleic acid reduces the hepatic triacylglycerol content and the leptin mRNA level in adipose tissue in obese Zucker fa/fa rats. Br J Nutr. 2009;102:803-15.

6. Clément L, Poirier H, Niot I, Bocher V, Guerre-Millo M, Krief S, Staels B, Besnard P. Dietary trans-10,cis-12 conjugated linoleic acid induces hyperinsulinemia and fatty liver in the mouse. J Lipid Res. 2002;43:1400-9.

7. Vyas D, Kadegowda AK, Erdman RA. Dietary conjugated linoleic acid and hepatic steatosis: species-specific effects on liver and adipose lipid metabolism and gene expression. J Nutr Metab. 2012;2012:932928.

8. Fassina P, Scherer Adami F, Terezinha Zani V, Kasper Machado IC, Garavaglia J, Quevedo Grave MT, Ramos R, Morelo Dal Bosco S. The effect of garcinia cambogia as coadjuvant in the weight loss process. Nutr Hosp. 2015;32:2400-8.

9. Sethi A. A review on "Garcinia cambogia - a weight controlling agent". Intern J Pharmceut Res Dev. 2011;3:13-24.

10. Kim KY, Lee HN, Kim YJ, Park T. Garcinia cambogia extract ameliorates visceral adiposity in C57BL/6J mice fed on a high-fat diet. Biosci Biotechnol Biochem. 2008;72:1772-80

11. Kim YJ, Choi MS, Park YB, Kim SR, Lee MK, Jung UJ. Garcinia Cambogia attenuates diet-induced adiposity but exacerbates hepatic collagen accumulation and inflammation. World J Gastroenterol. 2013;19:4689-701.

12. Dong Y, Cheng Z, Meng H, Liu H, Wu C, Khan AR. The effect of cultivar, sowing date and transplant location in field on bolting of welsh onion (Allium fistulosum L.). BMC Plant Biol. 2013:13:154.

13. Ford-Lloyd BV, Armstrong SJ. Welsh onion allium fistulosum L. In: Kalloo G, Bergh BO, editors. Genetic improvement of vegetable crops. London: Pergamon Press; 1993. p. 51-8.

14. Fritsch RM, Friesen N. Evolution, domestication, and taxonomy. In: Rabinowitch HD, Currah L. Allium crop science: recent advances. Wallingford, UK: CABI Publishing; 2002. p.5-30.

15. Vlase L, Parvu M, Parvu EA, Toiu A. Phytochemical analysis of Allium fistulosum L. and A. ursinum L. Dig J Nanomater Biostruct. 2013;8:457-67.

16. Chen $\mathrm{JH}$, Chen HI, Wang JS, Tsai SJ, Jen CJ. Effects of welsh onion extracts on human platelet function in vitro. Life Sci. 2000;66:1571-9.

17. Sang S, Lao A, Wang Y, Chin CK, Rosen RT, Ho CT. Antifungal constituents from the seeds of Allium fistulosum L. J Agric Food Chem. 2002;50:6318-21.

18. Yamamoto Y, Aoyama S, Hamaguchi N, Rhi GS. Antioxidative and antihypertensive effects of welsh onion on rats fed with a high-fat highsucrose diet. Biosci Biotechnol Biochem. 2005;69:1311-7.

19. Sung YY, Yoon T, Kim SJ, Yang WK, Kim HK. Anti-obesity activity of Allium fistulosum L. extract by down-regulation of the expression of lipogenic genes in high-fat diet-induced obese mice. Mol Med Rep. 2011;4:431-5.

20. Sung YY, Kim SH, Kim DS, Park SH, Yoo BW, Kim HK. Nutritional composition and anti-obesity effects of cereal bar containing Allium fistulosum (welsh onion) extract. J Funct Foods. 2014;6:428-37.

21. Kim DS, Kim SH, Cha J. Antiobesity effects of the combined plant extracts varying the combination ratio of Phyllostachys pubescens leaf extract and Scutellaria baicalensis root extract. Evid Based Complement Alternat Med. 2016;2016:9735276. 
22. Zhou JR, Li L, Pan W. Dietary soy and tea combinations for prevention of breast and prostate cancers by targeting metabolic syndrome elements in mice. Am J Clin Nutr. 2007;86:5882-8.

23. Sung YY, Kim DS, Kim SH, Kim HK. Anti-obesity activity, acute toxicity, and chemical constituents of aqueous and ethanol Viola mandshurica extracts. BMC Complement Altern Med. 2017;17:297.

24. Nimptsch K, Pischon T. Obesity biomarkers, metabolism and risk of cancer: an epidemiological perspective. Recent Results Cancer Res. 2016;208:199-217.

25. Alderete TL, Byrd-Williams CE, Toledo-Corral CM, Conti DV, Weigensberg M, Goran MI. Relationships between IGF-1 and IGFBP-1 and adiposity in obese African-American and Latino adolescents. Obesity (Silver Spring). 2010;19:933-8.

26. Krishan S, Richardson DR, Sahni S. Adenosine monophosphate-activated kinase and its key role in catabolism: structure, regulation, biological activity, and pharmacological activation. Mol Pharmacol. 2015;87:363-77.

27. Kahn BB, Alquier T, Carling D, Hardie DG. AMP-activated protein kinase: ancient energy gauge provides clues to modern understanding of metabolism. Cell Metab. 2005;1:15-25.

28. Park MY, Lee KS, Sung MK. Effects of dietary mulberry, Korean red ginseng, and banaba on glucose homeostasis in relation to PPAR-alpha, PPARgamma, and LPL mRNA expressions. Life Sci. 2005;77:3344-54.

29. Westphal S, Borucki K, Taneva E, Makarova R, Luley C. Adipokines and treatment with niacin. Metabolism. 2006:55:1283-5.

30. Andrews ZB, Erion DM, Beiler R, Choi CS, Shulman GI, Horvath TL. Uncoupling protein-2 decreases the lipogenic actions of ghrelin. Endocrinology. 2010;151:2078-86.

31. Chandran M, Phillips SA, Ciaraldi T, Henry RR. Adiponectin: more than just another fat cell hormone? Diabetes Care. 2003;26:2442-50.

32. Finelli C, Tarantino G. What is the role of adiponectin in obesity related non-alcoholic fatty liver disease? World J Gastroenterol. 2013;19:802-12.

33. Arita Y, Kihara S, Ouchi N, Takahashi M, Maeda K, Miyagawa J, Hotta K, Shimomura I, Nakamura T, Miyaoka K, Kuriyama H, Nishida M, Yamashita S, Okubo K, Matsubara K, Muraguchi M, Ohmoto Y, Funahashi T, Matsuzawa Y. Paradoxical decrease of an adipose-specific protein, adiponectin, in obesity. Biochem Biophys Res Commun. 1999;257:79-83.

34. Yatagai T, Nagasaka S, Taniguchi A, Fukushima M, Nakamura T, Kuroe A, Nakai Y, Ishibashi S. Hypoadiponectinemia is associated with visceral fat accumulation and insulin resistance in Japanese men with type 2 diabetes mellitus. Metabolism. 2003;52:1274-8.

35. Maffei M, Halaas J, Ravussin E, Pratley RE, Lee GH, Zhang Y, Fei H, Kim S, Lallone R, Ranganathan S, et al. (1995). Leptin levels in human and rodent: measurement of plasma leptin and Ob RNA in obese and weight-reduced subjects. Nat Med. 1995;1:1155-1161.

36. Seiva FR, Chuffa $L G$, Braga CP, Amorim JP, Fernandes AA. Quercetin ameliorates glucose and lipid metabolism and improves antioxidant status in postnatally monosodium glutamate-induced metabolic alterations. Food Chem Toxicol. 2012;50:3556-61.

37. Son MJ, Rico CW, Nam SH, Kang MY. Influence of oryzanol and ferulic acid on the lipid metabolism and antioxidative status in high fat-fed mice. J Clin Biochem Nutr. 2010;46:150-6.

38. Ahn J, Lee H, Kim S, Park J, Ha T. The anti-obesity effect of quercetin is mediated by the AMPK and MAPK signaling pathway. Biochem Biophys Res Commun. 2008:373:545-9.

39. Hussein MA. Potential mechanisms for the antioxidant effects of Jasonia Montana. J. Pharm. Res Rev. 2011;1:19-28

40. Kumari S, Deori M, Elancheran R, Kotoky J, Devi R. In vitro and in vivo antioxidant, anti-hyperlipidemic properties and chemical characterization of centella asiatica (I.) extract. Front Pharmacol. 2016;7:400

41. Rice-Evans CA, Miller NJ, Paganga G. Antioxidant properties of phenolic compounds. Trends Plant Sci. 1997;2:152-9.

42. Chen ZY, Chan PT, Ho KY, Fung KP, Wang J. Antioxidant activity of natural flavonoids is governed by number and location of their aromatic hydroxyl groups. Chem Phys Lipids. 1996;79:157-63.

43. Parvu M, Toiu A, Vlase L, Alina Parvu E. Determination of some polyphenolic compounds from allium species by HPLC-UV-MS. Nat Prod Res. 2010;24:1318-24.

\section{Submit your next manuscript to BioMed Central and we will help you at every step:}

- We accept pre-submission inquiries

- Our selector tool helps you to find the most relevant journal

- We provide round the clock customer support

- Convenient online submission

- Thorough peer review

- Inclusion in PubMed and all major indexing services

- Maximum visibility for your research

Submit your manuscript at www.biomedcentral.com/submit
Biomed Central 